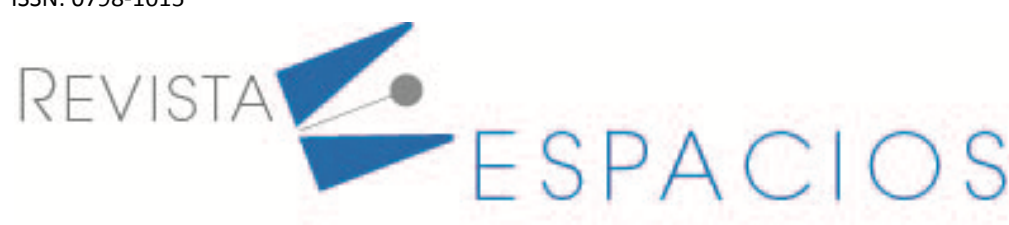

\title{
Aplicación móvil para apoyar el aprendizaje de estructuras de datos dinámicas utilizando realidad aumentada
}

\section{Mobile application to support the learning of dynamic data structures using augmented reality}

\author{
DÍAZ, Edgardo J. ${ }^{1}$ \\ FRANCO, David A. ${ }^{2}$ \\ MARTELO, Raúl J. ${ }^{3}$
}

\section{Resumen}

Este trabajo tuvo como objetivo brindar a los estudiantes de computación y áreas afines, una herramienta para facilitar el aprendizaje de estructuras de datos dinámicas, haciendo énfasis en listas enlazadas, esta se elaboró basada en una metodología aplicada, explicativa y mixta, resultando una Aplicación móvil y una cartilla que contiene los marcadores que permiten visualizar los objetos en realidad aumentada. Tras la implementación de la aplicación se concluyó que contribuye significativamente en el aprendizaje de listas enlazadas y su implementación.

Palabras clave: realidad aumentada, aplicación móvil, estructuras de datos dinámicas, listas enlazadas

\begin{abstract}
The objective of this work was to provide students of computing and related areas with a tool to facilitate the learning of dynamic data structures, emphasizing linked lists, this was developed based on an applied, explanatory and mixed methodology, resulting in a mobile application and a booklet containing markers that allow objects to be viewed in augmented reality. After the implementation of the app, it was concluded that it contributes significantly to the learning of linked lists and their implementation.
\end{abstract}

Key words: augmented reality, mobile application, dynamic data structures, linked lists

\section{Introducción}

Desde finales del siglo XX e inicios de este milenio se discutía sobre la importancia que cada día tomaba la tecnología para hacer de los procesos de aprendizaje más interactivos, con el uso del CD-ROM y páginas web para apoyar los procesos académicos, en general, se comenzó a dar una mayor apreciación de la interacción entre la tecnología y las actividades educativas (Vogel \& Klassen, 2001). Las instituciones educativas han invertido en mejorar sus plataformas tecnológicas, y con la aparición de la pandemia ocasionada por el COVID-19 se han visto obligadas a invertir mucho más. Los resultados generales del uso del e-learning para la evaluación formativa

\footnotetext{
${ }^{1}$ Ingeniero de Sistemas. Integrante del grupo de investigación GIMATICA. Programa de Ingeniería de Sistemas de la Universidad de Cartagena. e-mail: ediazp@unicartagena.edu.co

${ }^{2}$ Magister en Ciencias Computacionales. Líder del grupo de investigación GIMATICA. Profesor de planta del Programa Ingeniería de Sistemas de la Universidad de Cartagena. e-mail: dfrancob@unicartagena.edu.co

${ }^{3} \mathrm{PhD}(\mathrm{e})$ - Magister en Informática. Líder del grupo de investigación INGESINFO. Profesor de planta del Programa Ingeniería de Sistemas de la Universidad de Cartagena. e-mail: rmartelog1@unicartagena.edu.co
} 
han contribuido a los sistemas en línea para la enseñanza, el aprendizaje y la evaluación de los estudiantes puedan implementarse con mayor confianza (Oraifige, Heesom, \& Felton, 2009).

Investigadores de la Escuela de Ciencias de la Información del Instituto de Ciencia y Tecnología de Nara (Ikoma, Nara, Japón) y de la Escuela de Ingeniería y Ciencias de la Información del Instituto de Tecnología de Tokio (Meguro-ku, Tokio, Japón), realizaron una revisión de experiencias de aprendizaje en realidad aumentada aplicada en entornos educativos de preescolar, primaria y secundaria. El análisis realizado muestra que estas experiencias de aprendizaje lograron un efecto muy variable en el rendimiento de los estudiantes, pero resaltando que la realidad aumentada tiene tres ventajas inherentes: anotación del mundo real, visualización contextual y visualización háptica de la visión. Ilustraron estas ventajas a través de prototipos ejemplificadores, y basándose en la teoría del aprendizaje multimedia, la teoría del aprendizaje experiencial y la teoría de la visión animada (Santos, y otros, 2014).

En otra investigación realizada en España, muestra que los teléfonos inteligentes combinados con las tecnologías de realidad aumentada y las herramientas educativas como los Sistemas de Gestión de Aprendizaje (LMS), permiten incrementar los entornos informales y no formales para aumentar la continuidad natural entre el aprendizaje en todos los entornos, esa investigación contribuyó a proporcionar información sobre el diseño (perspectiva tecnológica) y la aplicación (perspectiva educativa) de las tecnologías de realidad aumentada en entornos informales y no formales con fines de aprendizaje formal (Pérez-Sanagustín, Hernández-Leo, Santos, Delgado Kloos, \& Blat, 2014).

VECAR es un aula virtual desarrollada en Taiwán para promover el aprendizaje del idioma inglés de manera inmersiva e interactiva, utilizando realidad aumentada y visión computacional. La inmersión físico-virtual y la interacción en tiempo real juegan un papel fundamental en el aprendizaje cultural y de idiomas. Los objetivos de VECAR fueron hacer que el aprendizaje cultural y de idiomas sea atractivo, mejorar la eficacia del aprendizaje cultural y mejorar la comunicación interpersonal entre profesores y estudiantes. Los resultados de la evaluación mostraron que el VECAR mejoró la efectividad del aprendizaje cultural y promovió la comunicación interpersonal entre profesores y estudiantes (Yang \& Liao, 2014).

En Italia, desarrollaron una versión avanzada de un simulador de realidad aumentada para colecistectomía laparoscópica, describiendo métodos de seguimiento para localizar el laparoscopio y las estructuras anatómicas tubulares. Además, presentaron una estrategia para reconstruir la forma de las estructuras triangulares de Calot, así como un procedimiento de calibración específico que permite mover libremente el laparoscopio manteniendo la coherencia geométrica de la escena en realidad aumentada. Pruebas preliminares mostraron que el simulador de realidad aumentada cumplió con todas las especificaciones iniciales: buena apariencia anatómica, modularidad, reutilización, minimización del costo de repuestos, robustez, capacidad para generar señalización acústica de posibles daños a las estructuras y proporcionar visualización de realidad aumentada para mostrar la posición de las estructuras triangulares de Calot (Viglialoro, y otros, 2019).

Cada día se aprecian nuevas aplicaciones para apoyar la enseñanza en distintas áreas del conocimiento, aplicando realidad aumentada para facilitar el aprendizaje de los estudiantes. En Italia desarrollaron una aplicación llamada Geo+, para apoyar el aprendizaje de geometría de niños de primaria, mostrando resultados de la eficacia del aprendizaje de los estudiantes, los niños apreciaron mucho la facilidad de uso del juego y se sintieron satisfechos y cómodos en la interacción con la aplicación, destacando que ninguno de ellos solicitó ayuda durante la ejecución de las actividades de aprendizaje (Rossano, Lanzilotti, Cazzolla, \& Roselli, 2020).

AR-SaBEr es una herramienta de simulación basada en realidad aumentada, para descubrir los principios básicos de la electricidad a través de una serie de experimentos, AR-SaBEr se mejoró con soporte basado en conocimientos y mecanismos de andamiaje basados en consultas, que resultaron útiles para el aprendizaje por 
descubrimiento en entornos de simulación basados en la web. Los factores de desempeño en el aprendizaje evaluados incluyeron el comportamiento de aprendizaje de los estudiantes mientras interactuaban con el sistema, la efectividad del aprendizaje de AR-SaBEr y la motivación de los estudiantes en el uso del entorno de simulación basado en realidad aumentada. El estudio realizado indicó que los simuladores de realidad aumentada pueden utilizarse como entornos de aprendizaje eficaces para aprender los principios básicos de la electricidad (Ibáñez, Di-Serio, Villarán-Molina, \& Delgado-Kloos, 2015).

En la Universidad de California se realizó un estudio fundamental de usuarios para evaluar los beneficios potenciales del uso de la tecnología de realidad aumentada para el aprendizaje de un vocabulario. Con la idea de que los sistemas de realidad aumentada pronto podrán etiquetar objetos del mundo real en cualquier idioma en tiempo real, el estudio de laboratorio se realizó con 52 personas, explorando el vocabulario en la aplicación de realidad aumentada para aprender sustantivos en un idioma extranjero desconocido, en comparación con un enfoque de aprendizaje tradicional basado en tarjetas. Los resultados mostraron que la experiencia en realidad aumentada de aprender con etiquetas virtuales en objetos del mundo real fue más efectiva y más agradable para la mayoría de los participantes del estudio, en comparación con las tarjetas tradicionales (Ibrahim, y otros, 2018).

En la Universidad de Khalifa (Abu Dhabi, Emiratos Árabes Unidos) desarrollaron un sistema móvil educativo basado en realidad aumentada, denominado AIR-EDUTECH, que fue realizado para ayudar a estudiantes de secundaria a aprender química. AIR-EDUTECH introdujo funcionalidades para ayudar a los estudiantes a comprender y aprender mejor los conceptos básicos de la química molecular, ofreciendo una visualización inmersiva en 3D e interacción visual con las estructuras examinadas proporcionando un conocimiento más amplio y retentivo, mejorando la intuición sobre la formación de reacciones químicas básicas. Los datos recopilados revelaron que AIR-EDUTECH estimuló mejoras significativas en la comprensión y retención del contenido enseñado y convirtió el aprendizaje de la química en una experiencia divertida, interesante e interactiva (Cen, Ruta, Al Qassem, \& Ng, 2020).

Investigadores de las Universidad Hamad Bin Khalifa (Doha, Qatar), de la Universidad Islámica Internacional de Malasia (Kuala Lumpur, Malasia) y de la Universidad de Malaya (Kuala Lumpur, Malasia) realizaron una revisión sistemática de estudios primarios relevantes sobre el uso de la realidad aumentada para mejorar diversas habilidades de niños y adolescentes diagnosticados con trastorno del espectro autista (TEA), desde los años 2005 a 2018 inclusive en ocho bases de datos bibliográficas. En la revisión abordaron once preguntas de investigación específicas relacionadas con las habilidades de aprendizaje, los participantes, la tecnología de realidad aumentada, el diseño de la investigación, los métodos de recopilación de datos, los entornos, los parámetros de evaluación, los resultados de la intervención, la generalización y el mantenimiento. Los resultados de los estudios mostraron que la realidad aumentada benefició a los niños con TEA en habilidades de aprendizaje (Khowaja, y otros, 2020).

El diseño de estructuras de datos dinámicas, específicamente de listas enlazadas y su implementación, son temas que generalmente son parte de la malla curricular de programas de formación profesional en ciencias de la computación y áreas afines, es uno de los temas que genera mayor dificultad a los estudiantes, porque demanda de habilidades para abstraer y entender el funcionamiento de los diferentes tipos de listas enlazadas y su relación con la gestión dinámica de la memoria. Los instructores o tutores en ocasiones deben hacer un mayor esfuerzo para exponer o ilustrar de forma gráfica estos conceptos, si los estudiantes tuviesen una herramienta que les ayudara a entender mejor estos conceptos, a los profesores se les facilitaría su trabajo y los estudiantes tuvieran un mejor rendimiento.

La aplicación se desarrolló para trabajar en teléfonos inteligentes bajo Android, empleando realidad aumentada ilustrando el funcionamiento de los distintos tipos de listas enlazadas. Como resultado se obtuvo una serie de 
animaciones que ilustran las operaciones que se realizan en una lista enlazada en utilización conjunta de una cartilla que contiene todos los temas a tratar y un teléfono inteligente con la aplicación terminada.

\section{Metodología}

El trabajo realizado corresponde a un tipo de investigación aplicada, explicativa y mixta, en la recolección de los datos fue necesario realizar una exploración exhaustiva de la bibliografía relacionada con el diseño e implementación de estructuras de datos dinámicas, se realizó investigación de campo para entrevistar profesores que han dirigido cursos relacionados con el tema, en el cual sus aportes fueron de gran importancia para identificar los requisitos de la aplicación y desarrollo del mismo, debido a sus experiencias y conocimiento del tema.

Muchos de los datos suministrados por los profesores fueron de gran ayuda para la elaboración del material ilustrativo, como la cartilla y los videos, donde se incluyeron las distintas operaciones (inserción, eliminación, búsqueda, visualización y destrucción) que se pueden realizar sobre las listas enlazadas fundamentales, para facilitar el aprendizaje del diseño e implementación de estructuras de datos dinámicas.

Posteriormente se elaboró la cartilla, que contiene información relacionada con la temática de listas enlazadas, su diseño e implementación en C++ (Standard C++ Foundation, 2020), incluyendo los marcadores necesarios para poder visualizar los elementos que construyen la realidad aumentada.

Finalmente, se elaboró la aplicación para teléfonos inteligentes basados en Android para facilitar el aprendizaje de estructuras de datos dinámicas, realizando las respectivas pruebas al software desarrollado y elaboración de la documentación de la aplicación, con la finalidad de comprobar el funcionamiento del mismo

Para el diseño e implementación de la aplicación móvil, se trabajó con una metodología mixta basada en la ingeniería de software basada en componentes (Vale, y otros, 2016) y AODDEI (Análisis, Obtención, Diseño, Desarrollo, Evaluación e Implementación) (Tovar, Bohórquez, \& Puello, 2014), esta propuesta metodológica fue la que mejor se adaptó a la investigación, aplicando sus fases se inició con el análisis del negocio, recolectando toda la información referente al tema de estructuras de datos dinámicas, en especial lo relacionado con listas enlazadas, definiendo los contenidos que hacen parte de toda la temática y la base conceptual de los Objetos Virtuales de Aprendizajes (OVAs).

En la etapa de diseño se realizaron los diagramas de casos de uso, diagrama de componentes y despliegue, definiendo la arquitectura de la aplicación y la interacción de cada uno de sus componentes, además de identificar las posibles herramientas de desarrollo, realizando el estudio que permitió escoger la herramienta de desarrollo que se ajustó mejor a las necesidades y limitaciones del proyecto.

En la etapa de construcción se realizó el desarrollo de la aplicación móvil y se adaptaron los componentes de ingeniería de software, se crearon animaciones con base a una serie de imágenes, además de generar los marcadores para aplicar la realidad aumentada y los correspondientes audios. Asimismo, se produjeron los materiales multimedia de la aplicación estableciendo la interfaz de los usuarios y la cartilla de apoyo para la enseñanza, conformada por toda la temática de listas enlazadas y los respectivos marcadores.

En la etapa final, una vez finalizado el desarrollo de la aplicación, se procedió a evaluarlo después de ponerlo en funcionamiento ante un grupo de estudiantes, que se brindaron como voluntarios para trabajar con la herramienta desarrollada, evaluando su funcionalidad y recibir la retroalimentación de su experiencia como usuarios.

Las etapas de la metodología mixta aplicada y las tareas realizadas en el proyecto en cada etapa se indican en la Tabla 1, dándose cumplimiento a cada uno de los objetivos planteados. 
Tabla 1

Etapas de la metodología mixta aplicada

\begin{tabular}{|c|c|}
\hline Etapa & Actividad realizada \\
\hline Análisis del problema & $\begin{array}{c}\text { Exploración de la información. } \\
\text { Identificación de requisitos. } \\
\text { Definición del OVA. }\end{array}$ \\
\hline Diseño de la solución & $\begin{array}{c}\text { Elaboración artefactos del software } \\
\text { Elección herramienta de desarrollo }\end{array}$ \\
\hline Desarrollo de la solución & $\begin{array}{c}\text { Desarrollo de la aplicación móvil. } \\
\text { Producción de animaciones. }\end{array}$ \\
& $\begin{array}{c}\text { Realización de marcadores. } \\
\text { Preparación de la guía o cartilla. }\end{array}$ \\
\hline Pruebas del prototipo y retroalimentación & $\begin{array}{c}\text { Puesta en funcionamiento del software. } \\
\text { Realización de la retroalimentación y } \\
\end{array}$ \\
& ajustes correspondientes. \\
\hline
\end{tabular}

Fuente: Autores

\section{Resultados}

Después de estudiar toda la temática concerniente a estructura de datos dinámicas, especialmente sobre listas enlazadas, recolectando de diferentes, destacando libros universitarios, páginas web especializadas y la guía de profesores que dirigen la asignatura de Estructuras de Datos, se procedió a describir el análisis de dominio (Ver Tabla 2), basado en los objetos virtuales de aprendizaje que se definió realizar.

Tabla 2

Descripción del análisis de dominio

\begin{tabular}{|c|l|}
\hline Nombres de OVAs & $\begin{array}{l}\text { Pila, Cola, Lista simplemente enlazada, lista circular, lista } \\
\text { doblemente enlazada. }\end{array}$ \\
\hline Descripción & $\begin{array}{l}\text { Involucra la combinación de los distintos tipos de } \\
\text { operación que se realizan en las diferentes clases de listas } \\
\text { enlazadas. }\end{array}$ \\
\hline $\begin{array}{c}\text { Nivel de formación que } \\
\text { debe tener el usuario }\end{array}$ & Estudiante de pregrado o superior. \\
\hline Perfil del estudiante & $\begin{array}{l}\text { Estudiantes de computación o carreras afines que incluyan } \\
\text { Estructuras de Datos como asignatura. }\end{array}$ \\
\hline Objetivo & $\begin{array}{l}\text { Apoyar el proceso de enseñanza/aprendizaje de } \\
\text { estructuras de datos }\end{array}$ \\
\hline Granularidad & $\begin{array}{l}\text { Los OVAs se clasificaron teniendo en cuenta cada } \\
\text { operación que se realizan en las estructuras de datos: } \\
\text { adición, borrado, búsqueda, visualización y destrucción. }\end{array}$ \\
\hline
\end{tabular}

Fuente: Autores

Después de realizar varias reuniones con profesores que dirigen o han dirigido la asignatura de Estructuras de Datos se establecieron los requisitos funcionales de la aplicación (Ver Tabla 3). 
Tabla 3

Requisitos funcionales de la aplicación

\begin{tabular}{|c|c|l|}
\hline ID & Nombre & \multicolumn{1}{c|}{ Descripción } \\
\hline R1 & $\begin{array}{c}\text { Identificar } \\
\text { marcadores }\end{array}$ & $\begin{array}{l}\text { La aplicación permitirá la identificación de marcadores } \\
\text { mediante la cámara del equipo o teléfono inteligente para } \\
\text { visualizar mayor información en imágenes 3D, videos, audio } \\
\text { o texto adicional. }\end{array}$ \\
\hline R2 & $\begin{array}{c}\text { Definir material } \\
\text { multimedia }\end{array}$ & $\begin{array}{l}\text { La aplicación incluirá material multimedia tales como } \\
\text { imágenes 3D, videos, audio, texto adicional, entre otros, } \\
\text { para apoyar la temática de estructuras de datos dinámicas. }\end{array}$ \\
\hline R3 & $\begin{array}{c}\text { Elaborar las } \\
\text { evaluaciones de cada } \\
\text { unidad temática. }\end{array}$ & $\begin{array}{l}\text { La aplicación tendrá un componente de evaluación para } \\
\text { cada clase de estructura de datos, con la finalidad de medir } \\
\text { la competencia alcanzada por el estudiante brindando la } \\
\text { retroalimentación respectiva. }\end{array}$ \\
\hline
\end{tabular}

Fuente: Autores

Al analizar varias herramientas para el desarrollo del software de realidad aumentada se decidió utilizar Vuforia (PTC Inc., 2020), que es una herramienta que acopla muy bien con la suite de Android, para el diseño y elaboración de las imágenes que describen la funcionalidad de las operaciones de las estructuras de datos dinámicas, se manejaron con Adobe Photoshop (Adobe Inc., 2020).

Para comprender la organización de las partes que debe contener el Objeto Virtual de Aprendizaje (OVA), deben cumplirse los requisitos indicados en la Tabla 4.

Tabla 4

Organización del Objeto

Virtual de Aprendizaje

\begin{tabular}{|c|l|}
\hline Objetivo & $\begin{array}{l}\text { Contribuir al proceso de enseñanza-aprendizaje de las estructuras de } \\
\text { datos dinámicas. }\end{array}$ \\
\hline $\begin{array}{c}\text { Contenido } \\
\text { informativo }\end{array}$ & $\begin{array}{l}\text { Los objetos virtuales de aprendizaje contienen apartados en los } \\
\text { cuales el estudiante pueda tener acceso a los contenidos } \\
\text { multimediales relacionados con la temática. }\end{array}$ \\
\hline Actividades & $\begin{array}{l}\text { El estudiante debe poder visualizar los videos e imágenes, y escuchar } \\
\text { los audios que realizan las explicaciones de las operaciones que se } \\
\text { permiten realizar en las estructuras de datos dinámicas. }\end{array}$ \\
\hline Evaluación & $\begin{array}{l}\text { El estudiante debe validar con el fin de medir el nivel de apropiación } \\
\text { de conocimiento por parte de los estudiantes, se realiza una } \\
\text { evaluación. }\end{array}$ \\
\hline
\end{tabular}

Fuente: Autores

Para el diseño de la aplicación se elaboraron varios artefactos en notación UML, como son el diagrama de casos de uso (Figura 1), el modelo de dominio del sistema (Figura 2), el diagrama de componentes de la aplicación (Figura 3) y el diagrama de secuencia (Figura 4). 
Figura 1

Diagrama de casos de uso

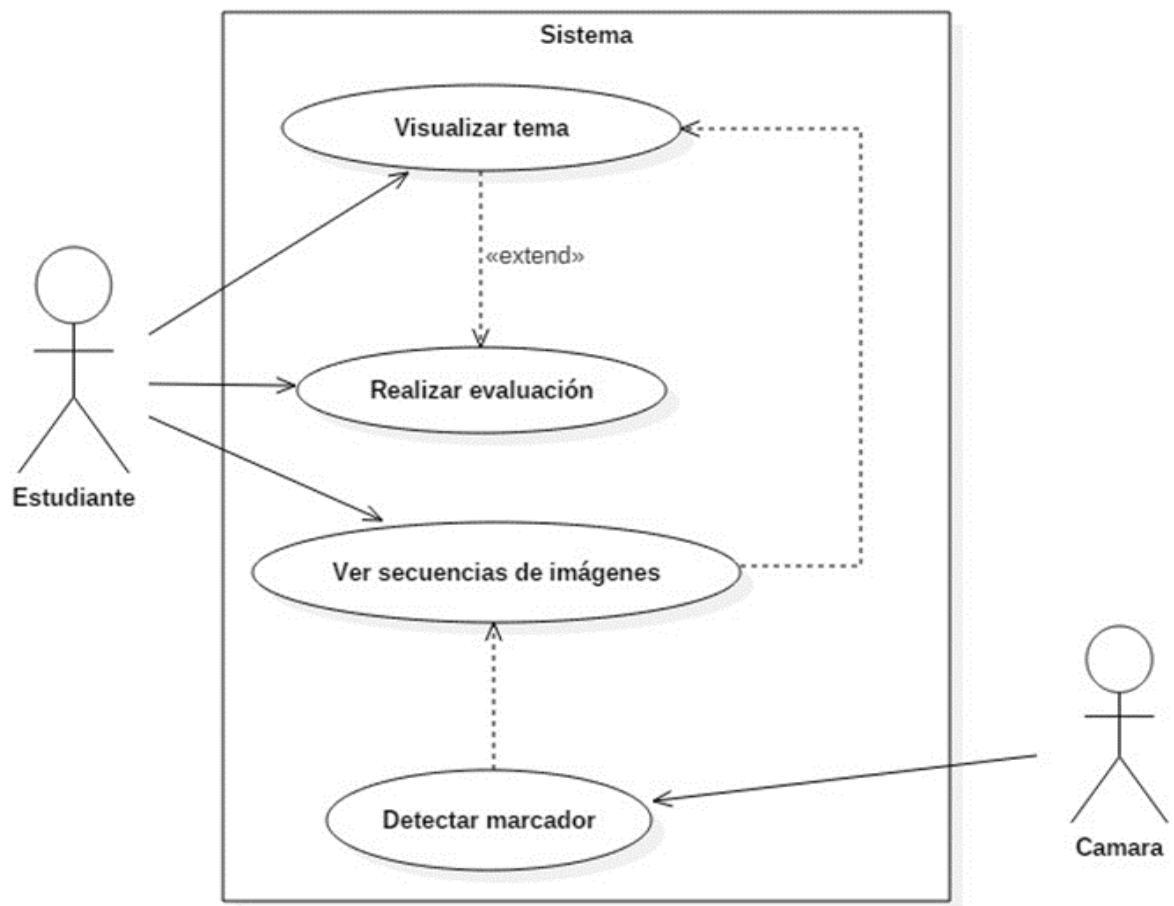

Fuente: Autores

Figura 2

Modelo de Dominio del sistema

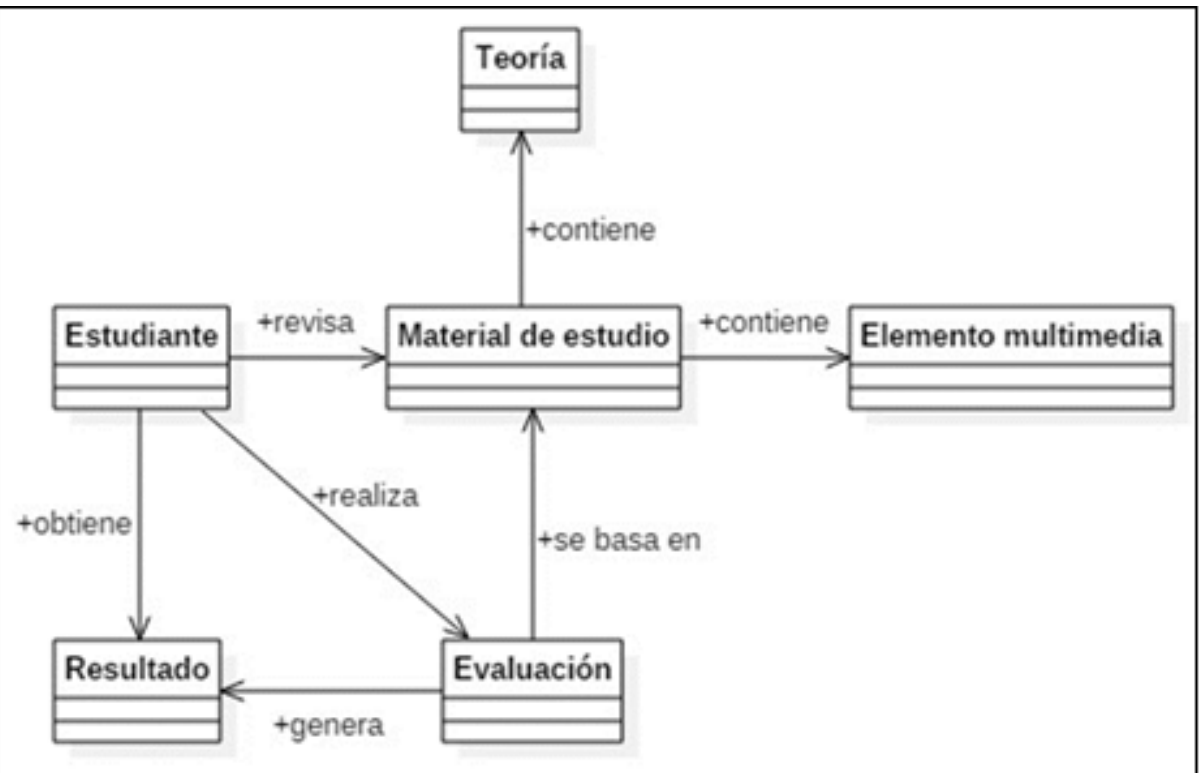

Fuente: Autores 
Figura 3

Diagrama de Componentes de la aplicación

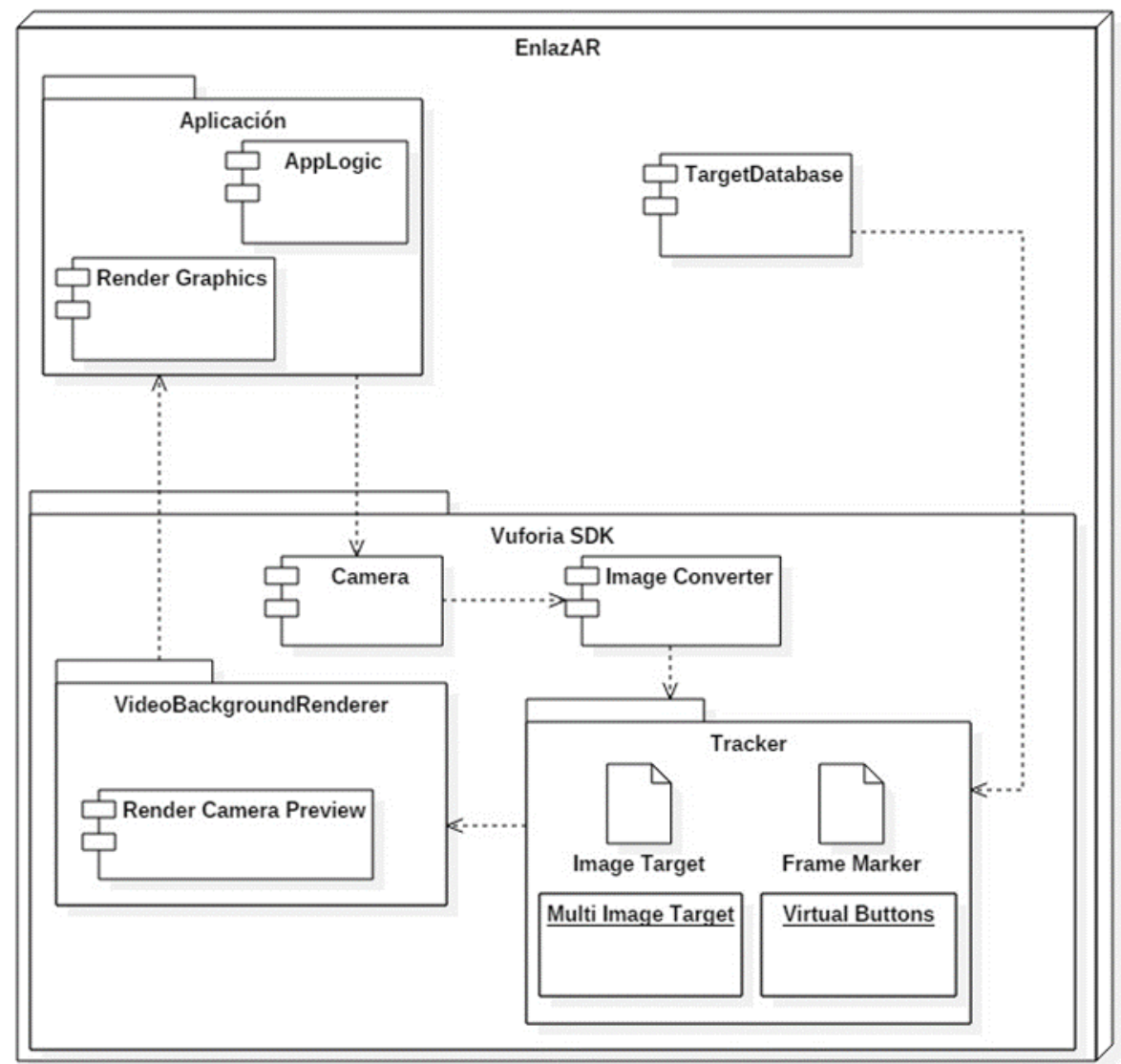

Fuente: Autores

Figura 4

Diagrama de Secuencia de la aplicación

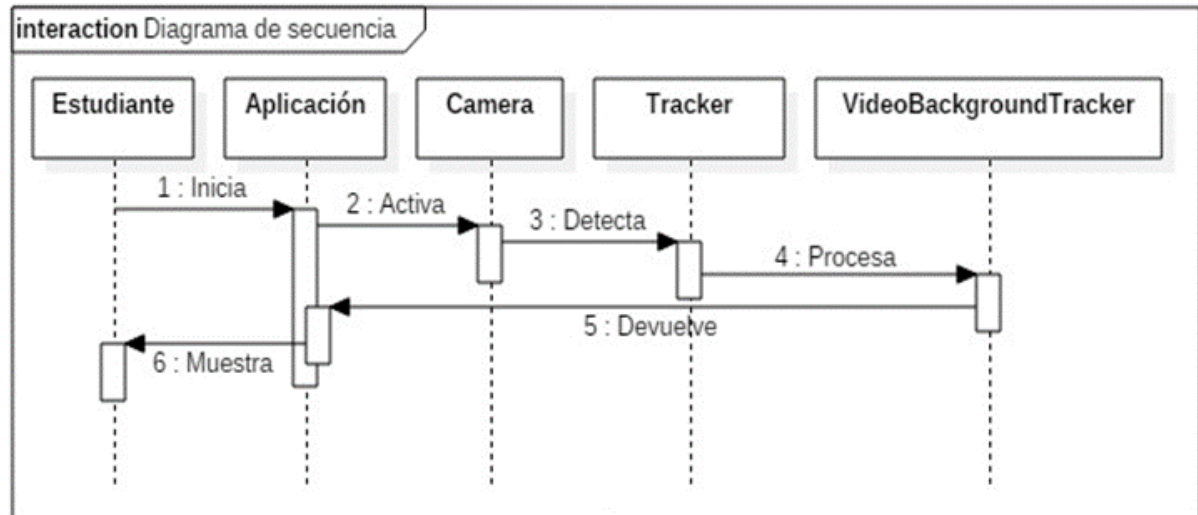

Fuente: Autores

Con la finalidad de realizar una explicación clara del funcionamiento de las estructuras de datos dinámicas y cada una de sus operaciones se generaron una serie de imágenes en la suite Adobe para ilustrar, paso a paso, como se afecta la estructura de datos respectiva y el código correspondiente para su implementación. En la Figura 5 se muestra una de esas imágenes. 
Figura 5

Una de las imágenes que ilustra la inserción

de un nodo en una lista simplemente enlazada

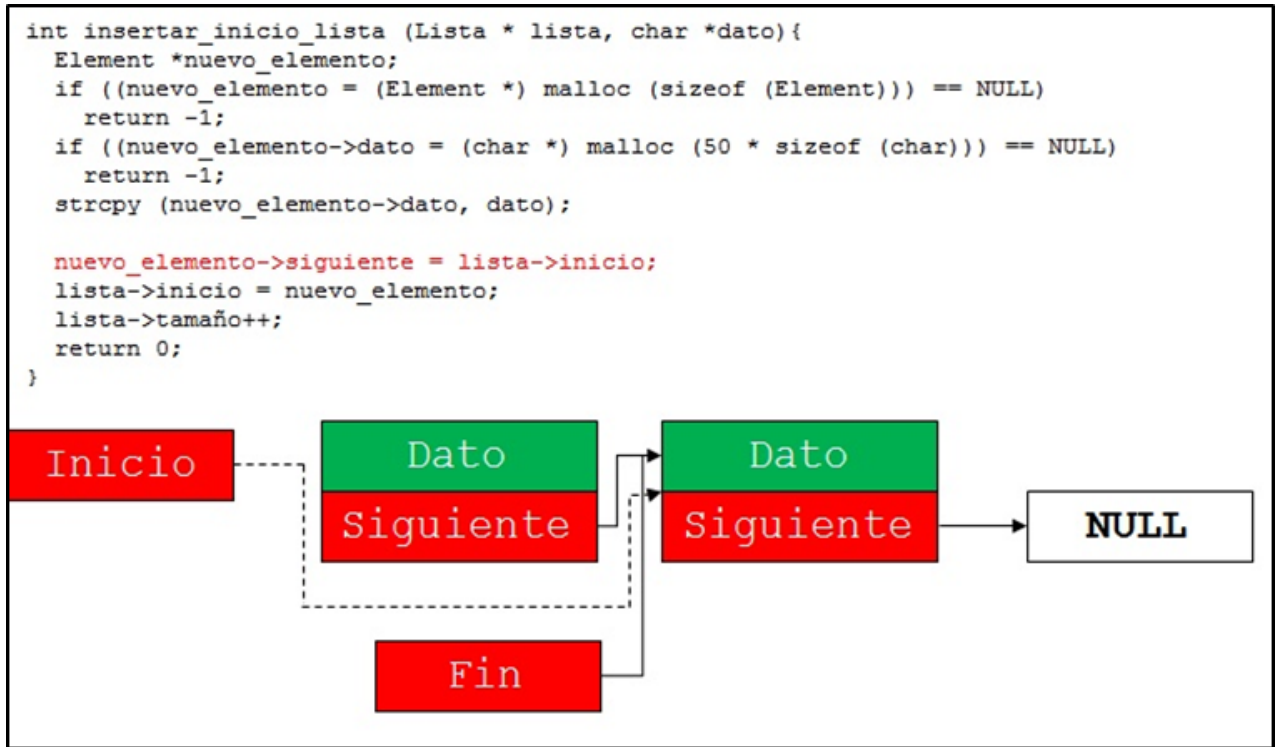

Fuente: Autores

En la generación del audio que acompaña cada explicación se empleó el software gratuito (freeware) Balabolka (Morozov, 2020), creando archivos de audio (mp3) desde un texto que se ingresa de parte del usuario. En la generación de marcadores, se creó un marcador inicial en el que se inserta texto con el fin de reconocer el tipo de estructura de datos y las instrucciones que se realizan sobre estas e impedir que los marcadores posean alguna semejanza, se manejó una aplicación web para la creación marcadores para realidad aumentada llamado Augmented Reality Marker Generator del fabricante Brosvision (Brosvision, 2020). Después de crear los marcadores de manera aleatoria, se crearon los diseños finales de los marcadores, quedando compuestos por el marcador inicial con un texto representativo, como se ilustra en la Figura 6.

Figura 6

Ejemplo de los marcadores utilizados

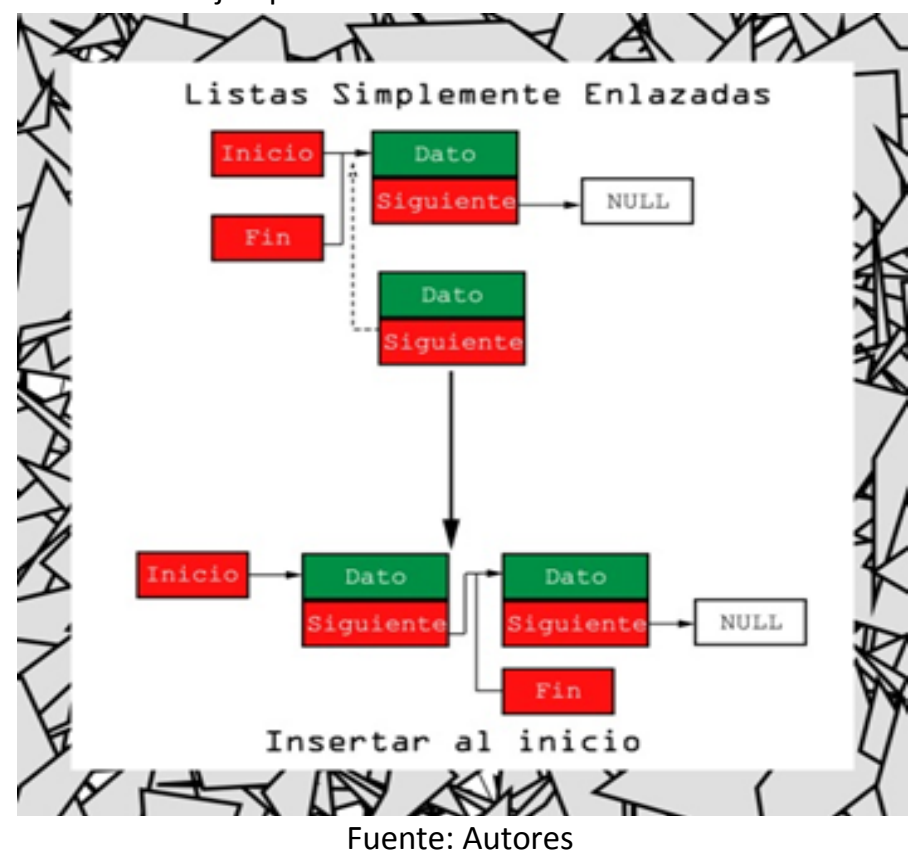


La construcción de la aplicación se realizó mediante código scripts en C\# dentro de Unity 3D y Javascript, que cumplieron con cada uno de los requisitos que se definieron al comienzo del proyecto. Con estos códigos y el SDK de Vuforia se logró generar la interfaz gráfica para los usuarios, y hacer parte de un sistema de apoyo para contribuir al proceso de enseñanza-aprendizaje de estructuras de datos dinámicas.

Figura 7

Percepción sobre la facilidad del uso de la aplicación

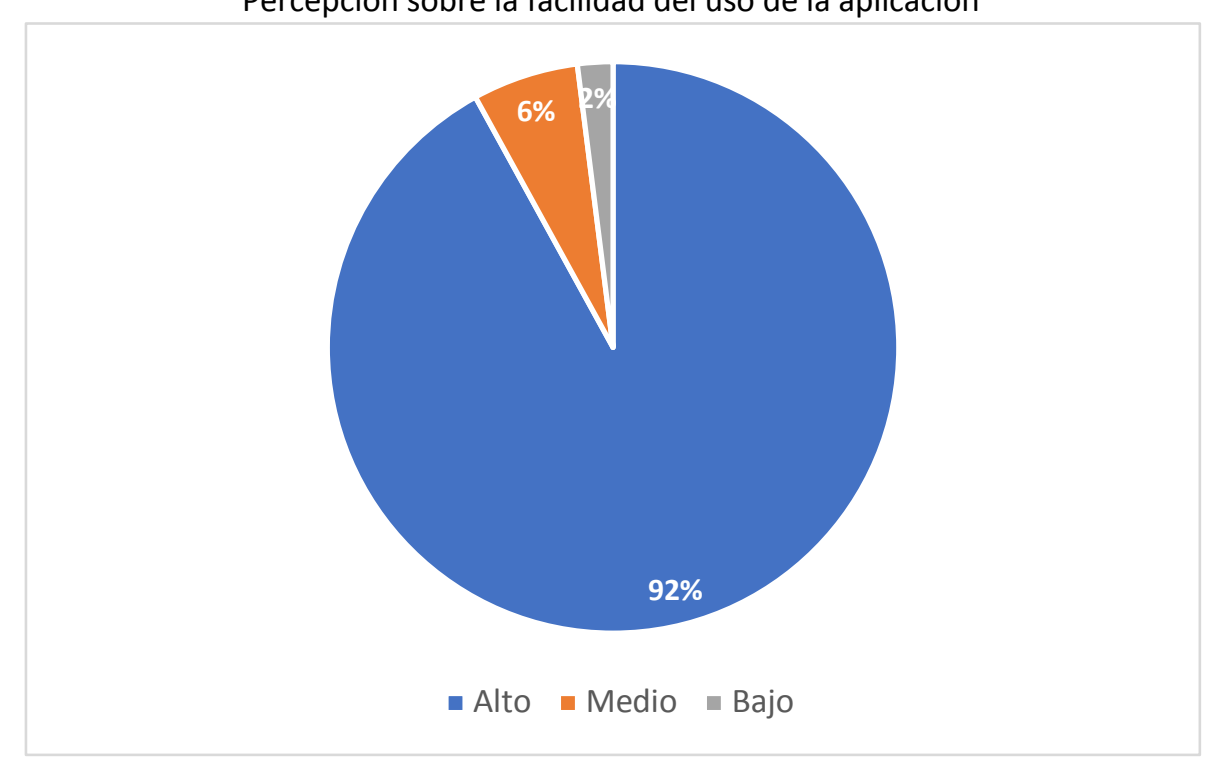

Fuente: Autores

En la etapa de prueba de la aplicación, se trabajó con un grupo de 33 estudiantes que cursaban la materia de Estructura de Datos, y también se probó con otro grupo de diez estudiantes que ya habían visto la materia, probándose en varios dispositivos, la mayoría teléfonos inteligentes, y algunas tabletas, todos con Android. Además de contar con la participación de tres docentes.

Figura 8

Percepción sobre la velocidad de la aplicación

\section{Velocidad de la aplicación}

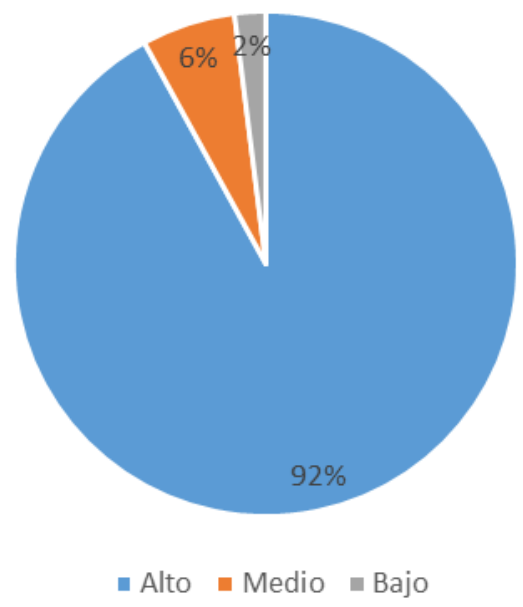

Fuente: Autores 
En la figura 7 se puede apreciar la percepción de los usuarios sobre la facilidad de uso de la aplicación desarrollada, indicando que el $92 \%$ de los usuarios les pareció que la aplicación tiene un nivel alto de facilidad de uso, esos mismos usuarios tuvieron una percepción equivalente sobre la velocidad de la aplicación (Ver Figura 8), es decir, que el tiempo de respuesta de la aplicación es satisfactoria, aclarando que el rendimiento se puede ver afectado un poco por las especificaciones técnicas del dispositivo.

Además, el $100 \%$ de los participantes de las pruebas coincidieron en que los contenidos manejados son pertinentes, completos y de gran ayuda al proceso de enseñanza aprendizaje.

\section{Conclusiones}

En este trabajo se desarrolló una aplicación basada en la tecnología de realidad aumentada para contribuir en el proceso de enseñanza-aprendizaje de estructuras de datos dinámicas, haciendo énfasis en los distintos tipos de listas enlazadas, que tradicionalmente hacen parte de los contenidos temáticos de la materia de Estructura de Datos en la mayoría de las universidades. Después de poner en funcionamiento la aplicación con un grupo de estudiantes y profesores, todos concluyeron que el sistema desarrollado maneja unos contenidos completos sobre la temática expuesta, destacando la facilidad de uso y un gran tiempo de respuesta al leer los marcadores para activar la realidad aumentada.

La aplicación final obtenida y la cartilla generada tienen información relacionada con la temática de estructuras de datos dinámicas, con el fin de que puedan ser utilizados de manera directa por los estudiantes interesados y aplicando también para los profesores que dirigen cursos relacionados con la temática, ofreciendo un alto grado de ilustraciones que contribuyen a una mejor captación de la atención de los estudiantes.

Durante la ejecución del proyecto se presentaron algunos inconvenientes en la lectura de los marcadores de realidad aumentada, debido a un error en el diseño inicial por tener los marcadores mucha similitud, que dificultaba su lectura, por lo que se diseñaron nuevamente mejorando su resolución y poder diferenciar los marcadores a simple vista.

En trabajos futuros se recomienda ampliar la temática a estructuras de datos no lineales, como son los árboles y grafos, con la finalidad de tener un sistema que integre todos los temas y cubra la totalidad del plan de estudios de la materia de Estructuras de Datos.

\section{Referencias bibliográficas}

Adobe Inc. (28 de 02 de 2020). Photo, Image \& Design - Adobe Photoshop. Obtenido de Adobe Photoshop: https://www.adobe.com/products/photoshop.html

Brosvision. (28 de 02 de 2020). Brosvision - Augmented Reality, Virtual Reality Development. Obtenido de Brosvision: https://www.brosvision.com/

Cen, L., Ruta, D., Al Qassem, L., \& Ng, J. (2020). Augmented Immersive Reality (AIR) for Improved Learning Performance: A Quantitative Evaluation. IEEE Transactions on Learning Technologies, 13(2), 283-296. doi:10.1109/TLT.2019.2937525

Ibáñez, M.-B., Di-Serio, Á., Villarán-Molina, D., \& Delgado-Kloos, C. (2015). Augmented Reality-Based Simulators as Discovery Learning Tools: An Empirical Study. IEEE Transactions on Education, 58(3), 208-213.

doi:10.1109/TE.2014.2379712 
Ibrahim, A., Huynh, B., Downey, J., Höllerer, T., Chun, D., \& O'donovan, J. (2018). ARbis Pictus: A Study of Vocabulary Learning with Augmented Reality. IEEE Transactions on Visualization and Computer Graphics, 24(11), 2867-2874. doi:10.1109/TVCG.2018.2868568

Khowaja, K., Banire, B., Al-Thani, D., Sqalli, M., Aqle, A., Shah, A., \& Salim, S. (2020). Augmented Reality for Learning of Children and Adolescents With Autism Spectrum Disorder (ASD): A Systematic Review. IEEE Access, 8, 78779-78807. doi:10.1109/ACCESS.2020.2986608

Morozov, I. (28 de 02 de 2020). Balabolka Versión: 2.15.0.756. Obtenido de Balabolka: http://www.cross-plusa.com/es/balabolka.htm

Oraifige, A., Heesom, D., \& Felton, A. (2009). Technology supported learning (TSL) for formative assessment. Engineering Education, 4(1), 61-67. doi:https://doi.org/10.11120/ened.2009.04010061

Pérez-Sanagustín, M., Hernández-Leo, D., Santos, P., Delgado Kloos, C., \& Blat, J. (2014). Augmenting Reality and Formality of Informal and Non-Formal Settings to Enhance Blended Learning. IEEE Transactions on Learning Technologies, 7(2), 118-131. doi:10.1109/TLT.2014.2312719

PTC Inc. (28 de 02 de 2020). Vuforia Developer Portal - Vuforia Engine 9.4. Obtenido de Vuforia Developer Portal: https://developer.vuforia.com/

Rossano, V., Lanzilotti, R., Cazzolla, A., \& Roselli, T. (2020). Augmented Reality to Support Geometry Learning. IEEE Access, 8, 107772-107780. doi:10.1109/ACCESS.2020.3000990

Santos, M., Chen , A., Taketomi, T., Yamamoto, G., Miyazaki, J., \& Kato, H. (2014). Augmented Reality Learning Experiences: Survey of Prototype Design and Evaluation. IEEE Transactions on Learning Technologies, 7(1), 38-56. doi:10.1109/TLT.2013.37

Standard C++ Foundation. (28 de 02 de 2020). Standar C++. Obtenido de Standar C++: https://isocpp.org/

Tovar, L., Bohórquez, J., \& Puello, P. (2014). Propuesta metodológica para la construcción de objetos virtuales de aprendizaje basados en realidad aumentada. Formación universitaria, 7(2), 11-20.

Vale, T., Crnkovic, I., Santana de Almeida, E., Silveira Neto, P., Cerqueira Cavalcanti, Y., \& Meira, S. (2016). Twenty-eight years of component-based software engineering. Journal of Systems and Software, 111, 128148. doi:https://doi.org/10.1016/j.jss.2015.09.019

Viglialoro, R., Esposito , N., Condino, S., Cutolo, F., Guadagni, S., Gesi, M., . . Ferrari, V. (2019). Augmented Reality to Improve Surgical Simulation: Lessons Learned Towards the Design of a Hybrid Laparoscopic Simulator for Cholecystectomy. IEEE Transactions on Biomedical Engineering, 66(7), 2091-2104. doi:10.1109/TBME.2018.2883816

Vogel, D., \& Klassen, J. (2001). Technology-supported learning: status, issues and trends. Journal of Computer Assisted Learning, 17(1), 104-114. doi:https://doi.org/10.1111/j.1365-2729.2001.00163.x

Yang, M.-T., \& Liao, W.-C. (2014). Computer-Assisted Culture Learning in an Online Augmented Reality Environment Based on Free-Hand Gesture Interaction. IEEE Transactions on Learning Technologies, 7(2), 107-117. doi:10.1109/TLT.2014.2307297

Esta obra está bajo una Licencia Creative Commons Attribución-NoCommercial 4.0 International

\section{$(\mathrm{Cc}) \mathbf{B Y}$-NC}

